\title{
APP PARA LA TERCERA EDAD: UTILIDAD, CLASES Y VALOR SOCIAL
}

\section{APP's FOR SENIORS: PROFIT, SOCIAL CLASSES AND VALUE}

Almudena Barrientos-Báez. Universidad de la Laguna. España.

David Caldevilla Domínguez. Universidad Complutense de Madrid, España.

Enrique García García. Universidad San Pablo CEU de Madrid, España.

\section{RESUMEN}

La proliferación de dispositivos inteligentes ha trascendido los ordenadores y los teléfonos móviles para pasar a integrarse progresivamente en todas partes: televisores, radios y ropa son o pronto serán inteligentes, susceptibles de nuevas aplicaciones y usos. Pero el logro más sorprendente de esta nueva tecnología es que, por primera vez, está estrechando la brecha tecnológica en vez de ensancharla: el público de la tercera edad está adoptando estos aparatos gracias a las facilidades de personalización y adaptación a sus necesidades específicas, tanto de accesibilidad como vitales. Nos hemos propuesto analizar este fenómeno y clasificar someramente las aplicaciones que lo han hecho posible, de manera que podamos entender mejor la importancia relativa de cada una.

PALABRAS CLAVE: brecha digital, APP, teléfonos inteligentes, tercera edad, mayores, interacción social, envejecimiento activo.

\section{ABSTRACT}

The widespread of smart-devices has gone further from computers \& phones, progressively integrating themselves everywhere: $t v$ is, radios and clothes are or shall be shortly inteligent, susceptible of new APP's and uses. But the most amaizing achievement has been, for the first time, the reduction of the digital breach between generations: the third age is adopting the new tech thanks to it is easiness in access and customization. We intend to analyze this phenomenon and to briefly classify those APP's that have made everything possible, so we can better understand the importance of each one.

KEY WORDS: digital breach, APPs, smartphones, old age, social interaction, active aging.

\section{Cómo citar el artículo:}

Barrientos-Báez, A., Caldevilla Domínguez, D. y García García, E. (2017). APP para la tercera edad: utilidad, clases y valor social. Revista de Ciencias de la Comunicación e Información, 22(2), 1-11.

doi: http://doi.org/10.35742/rcci.2017.22(2).1-11 


\section{INTRODUCCIÓN}

La sociedad actual está marcada por la evolución de la pirámide demográfica: debido al progresivo envejecimiento poblacional, la edad adulta se ha retrasado hasta etapas avanzadas de la vida, llevándonos a tener que definir de nuevo lo que supone la edad senil y el lugar temporal donde están situadas sus puertas (Parales y Dulce Ruiz, 2002; Lehr, 1980).

Beauvorianamente (Beauvour, 1983) madurez y vejez han dejado definitivamente de ser sinónimos. También resulta harto más complejo definir un intervalo temporal concreto que ataje el periodo de vejez (De Andrés y Maestro; 2014; Giró, 2004). Tampoco podemos aplicar la delimitación económica tradicional del periodo (Fericla, 2002; De Andrés y Maestro, 2014), con una esperanza de vida que pasa holgadamente de los 80 años, con todo lo que ello implica respecto a la edad "activa" es inequívocamente engañoso mantener que el momento del retiro sea aún indicativo del cambio de tercio vital. (Aldana, Fonseca y García Gómez, 2013).

Esta nueva realidad da importancia al también nuevo concepto del "envejecimiento activo". Según la OMS (2002) "el proceso de optimización de las oportunidades de salud, participación y seguridad para la mejora de la calidad de vida a medida que las personas envejecen". Este proceso, facilitado por las TIC, implica el progresivo avance hacia una sociedad sin brecha generacional tecnológica, con todos los potenciales beneficios que ello conlleva para la independencia individual, fomentando una participación social plena (Sánchez Martínez y Hatton-Yeo, 2012). De este modo, las nuevas tecnologías de la información adquieren si cabe mayor relevancia puesto que pasan a contribuir a la inclusión social y a la cohesión de sus miembros. (Plaza et al., 2011).

Las investigaciones que se han llevado a cabo en lo relativo a la relación entre TIC, sector móvil y mayores llevan a la conclusión de que es de la máxima importancia la contribución a la independencia de las personas de la tercera edad (Plaza et al. 2011). Este hecho ha motivado el diseño de las aplicaciones móviles para esos sectores de la población.

\section{OBJETIVOS}

Nos hemos propuesto observar las APP orientadas a este público del futuro, sobre todo en lo relativo a las redes sociales y la superación del aislamiento individual. Queremos saber qué APP se ofertan a ese público, y qué tipo de ayuda proporcionan con qué características destacables.

Para ello hemos explorado el software disponible para dispositivos inteligentes en Google Play, iTunes para los SO, iOS y Android, pues estas plataformas son aún las proveedoras más importantes de aplicaciones para dispositivos inteligentes. Todo se ha hecho mediante el uso de tags relacionados con el envejecimiento y la salud, fruto del preceptivo brain-storming (elderly, mayores, ancianos, teclado grande, tercera edad, silverhair, canas...) arrojando unos resultados totales (cribados los falsos positivos y las duplicidades) en torno a los 100 para Androis y 180 para iPhone. Analizando la información proporcionada por los desarrolladores, sus 
aptitudes y los requerimientos al dispositivo, se han clasificado en función de su utilidad.

\section{METODOLOGÍA}

\subsection{Relativas a la Salud}

Dentro de esta tipología hay, además, utilidades no exclusivas de uso para mayores. Aplicaciones que ayudan y aconsejan sobre dietética, aviso para no olvidar medicaciones importantes, adquisición de pertrechos o ayuda a domicilio, etc. No incluimos la evidente posibilidad de consultar como tratar algunas dolencias en el tiempo que tarda en llegar la ayuda, pero si las que permiten, por ejemplo, controlar constantes vitales del paciente. Una aplicación que a no mucho tardar, terminará estando disponible en ropa inteligente.

\subsection{Facilitadoras del Interface}

App de clara demanda destinadas a hacer más accesibles los terminales y su tecnología a las personas mayores: desde tonos de timbre más altos a teclados en pantalla con botones de mayor tamaño. Son las aplicaciones más fáciles de encontrar por cantidad de entradas (18\%).

\subsection{Diarias}

En definitiva, ayudas para garantizar la independencia de aquellos usuarios que viven solos (10\%) Pueden ser plataformas de contenidos con información sobre actividades que puedan ser del interés del usuario, o gestores del tiempo, libros digitales de cocina, aplicaciones de economía doméstica, o alarmas. Rodríguez Torres (2013) nos da el interesante ejemplo del colectivo de profesores y la doble tendencia que padece a aprovechar o ignorar las ventajas digitales para su labor.

\subsection{Seguridad}

El $13 \%$ de las APP revisadas versa sobre la seguridad: cámaras para que los familiares puedan seguir el día a día de los mayores cuando están solos; emergencia, destinadas mediante interfaces sencillos e inequívocos a alertar sobre necesidades urgentes de asistencia o necesidad y, finalmente, las de localización. Basadas en GPS para que ancianos perdidos o con afecciones cognitivas de algún tipo puedan ser localizados por la familia con rapidez.

\subsection{Entretenimiento}

El $18 \%$ de la muestra. Son aplicaciones muy sencillas de entender, dedicadas al tiempo de ocio: guías de turismo local, publicaciones digitales, juegos de psicomotricidad y agilidad mental... y aplicaciones sociales destinadas al contacto de adultos, normalmente ya solo con intención de que estos amplíen su red social real. 


\subsection{Comunicación interpersonal y Redes sociales}

Constituyen el $8 \%$ de la muestra, y van desde servicios de mensajería instantánea hasta aplicaciones de redes sociales generalistas o específicas, que permiten al usuario alejar el fantasma del aislamiento social, y acceder a nuevas formas de auto-realización y formación (Alonso García, 2013).

\section{DISCUSIÓN}

Estas tecnologías de la comunicación no han entrado en la sociedad sin producir efectos generacionales. A mayor edad, más bajos índices de adopción de las TIC (Cotten, 2011; File, 2013; Hsi-yi et al., 2014). En España, el 75\% de la población tiene y utiliza habitualmente internet; pero tan solo la cuarta parte de los mayores de 65 años han dado el salto a sus ventajas (INE, 2014) y la tendencia se radicaliza en lo relativo a los dispositivos inteligentes.

Es la llamada "Brecha digital" de la que se desprenden ideas preconcebidas sobre la relación entre TIC y personas mayores (Martínez, Cabecinhas y Loscertales, 2011). Lo que lleva a aquellos que entre ellos pueden considerarse digitalmente alfabetizados, a tener que enfrentarse a quienes asumen que no cuentan con la habilidad para manejar estos nuevos dispositivos, o que incluso los dan por tecnófobos. (Abascal y Civit, 2001).

Por eso mismo, la inclusión digital tiende a dejar fuera a los mayores, que deben enfrentarse la adquisición de habilidades y conocimiento con consideraciones diferentes al resto de la población: tales como diferente psicomotricidad o afecciones de síntomas aplicables (Hsi-yi et al, 2014; Seglers, Van Boxel y Jolles, 2009).

Su inclusión digital puede potenciarse gracias a políticas de alfabetización digital y al desarrollo de hardware y APP adecuadas a las necesidades específicas del grupo (Werner, 2011). Se ha acuñado el término "Gerotechnology" a causa de la creciente demanda de tecnologías adaptadas al presente y envejecido cuadro demográfico (Plaza et al., 2011).

Charnes, Parks y Sabel, (2001) hablan de las posibilidades que, una vez adaptadas, tienen estas nuevas tecnologías para este sector de la población. Le permite un más firme agarre a la realidad y actualidad social, abriendo el camino a convertir los "años dorados" en una etapa de continuado crecimiento (Aguado, Pascual y Fombona, 2013; Plaza et al., 2011) facilitando, por ejemplo, el establecimiento y mantenimiento de relaciones sociales mucho más allá de su entorno inmediato (Cornejo, Tentori, Favela, 2013). Estas suelen limitarse durante la vejez a raíz de la salida de grupos de pertenencia, la movilidad reducida e incluso las barreras arquitectónicas. Ahora es posible, sin embargo, estrechar relaciones sociales existentes y crear otras nuevas incluso a edades muy avanzadas (Agudo, Pascual y Fombona, 2013; Martínez Cabecinhas y Loscertales, 2011).

Donde Putnam preveyó una "crisis de comunidad" (1995) las TIC imponen el fin del aislamiento, herramientas en pro de la auto-imagen, y mejora de la calidad de vida a través del desarrollo de nuevas pericias (Hsin-yi et al., 2014; Amichai- Hamburger y 
Schenider, 2014). Considerando el concepto de gerotecnología como el aprovechamiento y orientación de las TIC para salvaguardar la salud biológica, mental y social (Plaza et al., 2011; Harrington y Harrington, 2000), los dispositivos móviles pasan a ser de una importancia ejemplar en cuanto a este estado de cosas, a causa de la difusión global del dispositivo inteligente y la forma en que ello mismo ha motivado la aparición de software específico para la población mayor.

\subsection{No es un teléfono: es un ordenador de bolsillo}

El móvil también ha entrado en las casas y en las chaquetas de la tercera edad (Kurniawan, 2007). El $61 \%$ de la población mayor de 65 años en el mundo desarrollado emplea el móvil de forma habitual (Fondo de Población de Naciones Unidas, 2012). El doble de los que utilizan internet y que para Plaza et al. (2011) se explica por motivos de seguridad personal, además de lo evidente (Oksman, 2006; Conci, 2007; Ling, 2008).

La adopción de estas tecnologías por parte de las personas mayores depende de que satisfagan sus necesidades (Phiriyapokanon, 2011). Esta población sabe de las ventajas potenciales, pero un exceso de dificultad o percepción de la misma puede llevarles a rechazarla (Czaja y Lee, 2007). Sin embargo, la difusión de las mismas ha llegado de manera casi completa incluso al significativo estereotipo que representa la tercera edad rural (Matellanes-Lazo, 2015) como, por otro lado, dicha población se sumó a la tecnología televisiva en su momento aunque esta implicara menos beneficios sociales (Olmo López, 2015).

Envejecer tiene como consecuencia una serie de trabas en lo relativo a la percepción sensorial, psicomotricidad reducida, etc... (Phiriyapokanon, 2011). Kurniawan (2007) difiere cuatro problemas de accesibilidad a las TIC para los mayores: tamaño y funcionalidad del interface, complejidad del interface, dimensiones del hardware y tamaño de texto. Problemas que nos nuevos dispositivos inteligentes aún tienen que resolver (Zhou et al., 2014).

El I-Phone (2007) modificó sensiblemente las ideas existentes sobre telefonía inteligente. Ni teléfonos ni ordenadores, se trata desde entonces más bien de pantallas dotadas de funciones: todas las funciones, accesibles gracias a multiples programas o aplicaciones que permiten un grado absoluto de personalización (Coelho et al, 2012; Phiriyapokanon, 2011; Czaja y Lee, 2007). La clave del futuro está en que esa personalización alcance cada vez más a sectores de la población excluidos de la revolución digital (Hsin-yi et al., 2013).

Hay numerosas vías de aproximación al problema, empezando por aquellas que tratan las aplicaciones relacionadas con el cuidado de la salud (Charnes, 2014, Gao y Koronios, 2010, Plaza et al., 2010 y Blake, 2008, Yu, Li y Gagnon 2009) sobre información de salud, de McGree-Lennon (2008) etc.

Pedell et al. (2013) o Drobics y Smith (2014) enfocan a los casual games por sus ventajas de potenciación cognitiva y psicomotriz, y -claro- por el entretenimiento que proporcionan. El ámbito de las aplicaciones con utilidad social no ha sido tan ampliamente estudiado. Pero Williams, Ahamed y Chu (2014) y Burkahard y Koch 
(2012) han hecho aportaciones valiosas respecto al diseño de tal software. Las aplicaciones de este tipo potencian la relación social de este segmento de la población, creando relaciones de interdependencia entre generaciones, haciendo que la OMS recomendara su uso (2002) por el potencial de corrección de desequilibrios sociales y personales que comportan.

\subsection{Comunicación en red: las redes sociales}

Una red social bien asentada, y entendida en su sentido analógico es objetivamente positiva para la calidad de vida de ancianos y jóvenes. De esta afirmación se trasluce la importancia real que las redes sociales digitales pueden tener en el mantenimiento de las analógicas. La adopción de estos métodos y tecnologías 2.0 por parte de la tercera edad conlleva también un objetivo social común a medio plazo (Vilte et al., 2013; Bell et al., 2013).

La población de mayor edad mira a las familias a la hora de establecer redes sociales (Bell et al., 2013; Cagley y Lee, 2009). Esto no es un obstáculo para la adopción del entorno 2.0, ya que contactar con familiares de residencia remota o durante viajes lejanos puede suponer una motivación capital (Vilte et al., 2013).

Si bien las plataformas sociales fueron concebidas para usuarios jóvenes (Vilte et al., 2013), tornándolas en ejemplos paradigmáticos de la brecha digital, los usuarios mayores se han abierto su propio espacio en ellas (Bell et al., 2013).

El Pew Research Centre (2014) fue el primero en apreciar un aumento del número de usuarios de edad madura entre los perfiles de Facebook, (en torno al 50\% de los internautas estadounidenses) para dicha edad.

Las personas mayores participan cada vez más en redes sociales, con cifras, eso sí, aún demasiado reducidas. Con 20 Millones de perfiles de Facebook en España, apenas el 3,5\% (700.000) son de internautas por encima de los 65 (FacebookAds, 2014). Marginando esto a buena parte de la tercera edad española de los social media y sus útiles aplicaciones en el fortalecimiento de redes de relaciones (Cornejo, Tentori y Favela, 2013).

\section{CONCLUSIONES}

El envejecimiento activo se ha vuelto una realidad un poco más palpable gracias a las TIC y a la movilidad de los dispositivos. Su utilidad objetiva y la posibilidad de alta personalización están consiguiendo lo imposible: estrechar la brecha tecnológica digital que parecía estar volviéndose insalvable. Los dispositivos inteligentes se han abierto paso hasta los usuarios de mayor edad, poniendo a su alcance todas las mejoras vitales que pueden ofrecer, y no tan solo en sentido figurado, posibilista, sino de una manera real y palpable.

Estas aplicaciones facilitadoras pueden clasificarse con facilidad en función de su finalidad y servicio que ofertan, y se distinguen por estar enfocadas hacia este público y sus necesidades de accesibilidad, sin que ello sea óbice para que aplicaciones como, por ejemplo, las que buscan de una manera u otra cuidar la salud del usuario, tengan un mayor nivel de complejidad en sus interfaces. 
Quizá la mayor aportación de estos dispositivos a la tercera edad sea la de ayudarla a consolidar sus redes sociales y a crear otras nuevas. El fantasma de la soledad de los mayores se aleja un poco gracias a este software. Las aplicaciones de comunicación permiten también un nivel de comunicación interfamiliar que obra en beneficio de la tranquilidad de todos los afectados, al mismo tiempo que evita la exclusión del colectivo.

Este acceso a redes sociales digitales puede llevar a la formación de comunidades específicas en torno a aplicaciones especializadas. Algo positivo siempre que no desemboque en comunidades digitales paralelas que alejen a sus usuarios de las redes generalistas, lo que, en definitiva, nos llevaría de nuevo al problema del aislamiento colectivo de estos usuarios. Un esfuerzo para acercar las redes generalistas a los usuarios de edad madura sería el impulso necesario en ese sentido. Para ello, lo más sencillo es, claro, generar una interrelación entre las redes específicas y las generalistas.

\section{REFERENCIAS}

Abascal, J. \& Civit, A. (2000). Mobile communication for people with disabilities and older people: New opportunities for autonomous life. En Proceedings of the 6th ERCIM Workshop. Florencia: ERCIM, 255-268. Recuperado de http://google/cHG9KX

Agudo, S.; Pascual, M. A. y Fombona, J. (2012). Usos de las herramientas digitales entre las personas mayores. Comunicar, 39(29), 193-201.

Aldana, G.; Fonseca, C. y García, L. (2013). El significado de la vejez y su relación con la salud en ancianas y ancianos integrados a un programa de envejecimiento activo. Revista Digital Universitaria, 14(4). Recuperado de http://google/VPgGLc

Amichai-Hamburguer, Y. \& Ben-Artzi, E. (2003). Loneliness and Internet use. Computers in human behavior, 19(1), 71-80.

Beavoir, S. de (1983). La vejez. Barcelona: Edhasa.

Bell, C.; Fausset, C.; Farmer, S.; Nguyen, J.; Harley, L. \& Fain, W. B. (2013). Examining social media use among older adults. En Proceedings of the 24th ACM Conference on Hypertext and Social Media, 158-163. AMC: New York.

Blake, H. (2008). Innovation in practice: mobile phone technology in patient care. British journal of community nursing, 13(4), 160-165.

Burkhard, M. \& Koch, M. (2012). Social Interaction Screen. Making Social Networking Services Accessible for Elderly People. i-com Zeitschrift für interaktive und kooperative Medien, 11(3), 3-7. Recuperado de http://google/83QE3s

Cagley, M. \& Lee, M. (2009). Social support, networks, and happiness. Today's Research on Aging: Program and Policy Implications, 17. Washington: Population Reference Bureau. 
Charness, N. (2014). Utilizing technology to improve older adult health. In Occupational therapy in health care, 28(1), 21-30.

Charness, N.; Parks, D. C. \& Sabel, B. A. (2001). Communication, technology and aging: Opportunities and challenges for the future. New York: Springer Publishing Company.

Coelho, J.; et al. (2012). Involving All Stakeholders in the Development of TV Applications for Elderly. ThinkMind. International Journal On Advances in Intelligent Systems, vol. 5, 3-4. Recuperado de http://google/Hr2Cdm

Conci, M. (2007). Mobile Phone Acceptance by Older People. Building and Validation of a theoretical model. Tesis doctoral. Trento: Universita Degli Studi di Trento.

Conde, M. Á., García-Peñalvo, F. J., \& Matellán-Olivera, V. (2014). Mobile apps repository for older people. En Proceedings of the Second International Conference on Technological Ecosystems for Enhancing Multiculturality. (pp. 725731). ACM: New York.

Cornejo, R.; Tentori, M. \& Favela, J. (2013). Enriching in-person encounters through social media: A study on family connectedness for the elderly. Journal of HumanComputer Studies, 71, 889-899.

Cotten, S. R. (2011). Overcoming digital divides in assisted and independent living communities: Results from a randomized trial. Gerontologist, 51, 220-230.

Czaja, S. \& Lee, C. (2007). The impact of aging on access to technology. Universal Access in the Information Society, 5(4), 341-349.

Del Campo, A. y Lima Maestro, R. D. (2014). Análisis crítico del discurso publicitario institucional/comercial sobre las personas mayores en España. Comunicar, 42(20), 189-197.

Drobics, M. \& Smith, S. (2014). Game-Based IT Solutions for Active and Healthy Aging.

Fercgla, J. E. (1992). Una antropología de la ancianidad. Barcelona: Anthropos.

File, T. (2013). Computer and internet use in the United States. Population Characteristics. Consultado el 10 de marzo de 2015. Recuperado de http://www.census.gov/prod/2013pubs/p20- 569.pdf

Gao, J. \& Koronios, A. (2010). Mobile Application Development for Senior Citizens. Consultado el 10 de marzo de 2015. Recuperado de http://google/nwnpCZ

Gao, J. \& Koronios, A. (2010). Mobile application development for senior citizens. Recuperado de http://www.pacis- net.org/file/2010/S05-03.pdf 
García, Alonso; et al. (2013). Las redes sociales en las universidades españolas, Revista de Comunicación de la SEECI. Año XVI (33).

Giró, J. (2004). El significado de la vejez. In Autor (Coord.), Envejecimiento y sociedad. Una perspectiva pluridisciplinar, 19- 46. Logroño: Universidad de la Rioja.

Gobel, S. \& Wiemeyer, J. (Eds.) Games for Training, Education, Health and Sports. 4th International Conference on Serious Games, p. 8-11. Springer International Publishing: Darmstadt.

Hampton, K. N. \& Gupta, N. (2008). Community and social interaction in the wireless city: wi-fi use in public and semi-public spaces. New Media \& Society, 10(6), 831850.

Harrington, T. L. \& Harrington, M. K. (2000). Gerontechnology: Why and how. The Netherlands: Shaker

Kurniawan, S. (2008). Older people and mobile phones: A multi-method investigation. International Journal of Human-Computer Studies, 66(12), 889-901.

Lehr, U. (1980). Psicología de la senectud. Barcelona: Herder.

Ling, R. (2008). Exclusion or self-isolation? Texting and the elderly users. The information society, 24(5), 334-341.

Martínez, R.; Cabecinhas, R. y Loscertales, F. (2011). Mayores universitarios en la Red. Comunicar, 37(20), 89-95.

Matellanes-Lazo, M. (2015). Uso de las nuevas tecnologías por las personas mayores en una localidad rural. Revista de Comunicación Vivat Academia, Año XVIII, 130.

McGee-Lennon, M. R. (2008). Requirements engineering for home care technology. In Proceedings of the SIGCHI Conference on Human Factors in Computing Systems. Ney York: ACM; pp. 1439-1442.

Oksman, V. (2006). Young people and seniors in Finnish 'mobile information society'. Journal of Interactive Media in Education, 2(1). Recuperado de http://jime.open.ac.uk/articles/10.5334/2006-3/.

Olmo-López, A. (2015). Revista de Comunicación Vivat Academia XVIII, №130.

Parales, C. J. y Dulcey, E. (2002). La construcción social del envejecimiento y de la vejez: un análisis discursivo en prensa escrita. Revista Latinoamericana de Psicología 34(1/2), 107-121.

Pedell, S.; Beh, J.; Mozuna, K. \& Duong, S. (2013). Engaging older adults in activity group settings playing games on touch tablets. In Proceedings of the 25th 
Australian Computer-Human Interaction Conference: Augmentation, Application, Innovation, Collaboration, pp. 477-480. ACM: New York.

Pew research center (2014). Social networking fact sheet. Recuperado de http://www.pewinternet.org/fact-sheets/social- networking-fact-sheet/ .

Piriyapokanon, T (2011). Is a Big Button Interface Enough for Elderly Users?: Towards user interface guidelines for elderly users. Recuperado de www.idt.mdh.se/examensarbete/index.php?choice=show\&id=1091

Plaza, I.; Martín, L.; Martín, S. \& Medrano, C. (2011). Mobile applications in an aging society: Status and trends. Journal of Systems and Software, 84(11), 1977- 1988.

Putnam, R. D. (1995). Bowling Alone: America's Declining Social Capital. Journal of Democracy, vol. 6, 65-78.

Rodríguez-Torres, J. (2013). Encrucijada docente: la inclusión de las tecnologías de la información y comunicación. Revista de Comunicación de la SEECI. Año XVII (31).

Sánchez, M. \& Hatton-Yeo, A. (2012). Active Ageing and Intergenerational Solidarity in Europe: A Conceptual Reappraisal from a Critical Perspective. Journal of Intergenerational Relationships, 10(3), 276-293.

Slegers, K.; Van Boxtel, M. \& Jolles, J. (2009). Effects of computer training and internet usage on cognitive abilities in older adults: a randomized controlled study. Aging clinical and experimental research, 21(1), 43-54.

Valenzuela, S.; Park, N. \& Kee, K. F. (2008). Lessons from Facebook: The effect of social network sites on college students' social capital. En 9th International Symposium on Online Journalism. Austin: Texas. Recuperado de http://google/ZY1pgy

Vilte, D.; Saldaño, V.; Martín, A. y Gaetán, G. (2013). Evaluación del uso de redes sociales en la tercera edad. Recuperado de http://google/UhrGHY

Werner, C. A. (2011). The older generation: 2010. U.S. Census Bureau. Recuperado de http://google/8qOapU

Williams, D.; Ahamed, S. I. \& Chu, W. (2014). Designing Interpersonal Communication Software for the Abilities of Elderly Users. En Computer Software and Applications Conference Workshops, pp. 282-287.IEEE 38th International. IEEE: Vasteras.

Yu, P.; Li, H. \& Gagnon, M. P. (2009). Health IT acceptance factors in long-term care facilities: a cross-sectional survey. International Journal of Medical Informatics, 78(4), 219-229. 
Zhang, S.; Jiang, H. \& Carroll, J. M. (2011). Integrating online and offline community through Facebook. En Collaboration Technologies and Systems (CTS), 2011 International Conference on Technologies and systems, pp. 569- 578. IEEE: Philadelphia.

Zhou, J.; Pei-Luen, P. R. \& Salvendy, G. (2014). Older adults text entry on smartphones and tablets. Investigating effects of display size and imput method on aceptance and performance. International Journal of Human Computer Interaction, 30(9), 727-739. 